\title{
Phonosurgical Training in India: A Sample Survey
}

\author{
Shaoni D Sanyal ${ }^{1}$, Amitabha Roychowdhury ${ }^{2}$, Ranjan Raychowdhury ${ }^{3}$
}

\begin{abstract}
Aim:The objective of this study was to assess exposure of postgraduate trainees in otolaryngology to phonosurgery in various colleges across India Materials and methods: A questionnaire was distributed to residents attending the 15th Annual Conference of the Association of Phonosurgeons of India (APSI) held at Kolkata in 2019. No personal or residency program identifying information was collected. The e-mail contained a hyperlink to the online survey. Data were extracted from online survey forms and processed by Microsoft Excel.

Results: Our study shows that of the 67 respondents, only 3 had access to all the necessary infrastructure [speech and language therapist (SLT), stroboscopy unit, and multidisciplinary voice clinic] required to treat patients. Fifty-seven respondents did not have a stroboscopy unit at their institution. The exposure to phonosurgery was restricted with only 25 respondents having a case load of more than 10 microscopic laryngeal surgeries (MLSs) per month. In our study, only $18 \%$ of the trainees were able to follow-up patients for a period of 6 months. Most trainees (55\%) in our study had performed MLS under supervision, and only $12 \%$ were able to perform it independently.

Conclusion: Our survey shows that of the 181 departments which offer masters as well as diploma in ENT in India, few are fully equipped to offer modern phonosurgical assessment and management, as a result of which very few trainees have adequate exposure in this subspeciality. Clinical significance: Few ENT departments in India are fully equipped to offer modern phonosurgical training. Workshops and cadaver dissections may be an effective way to impart better understanding of this field.

Keywords: Otolaryngology, Phonosurgery, Survey, Trainees.

International Journal of Phonosurgery \& Laryngology (2019): 10.5005/jp-journals-10023-1166
\end{abstract}

\section{InTRODUCTION}

Phonosurgery as a specialization is a relatively new offshoot of otorhinolaryngology.

The International Association of Phonosurgeons was established in 1990 in Belgrade. In the Indian context, Association of Phonosurgeons of India (APSI) was set up on 8th November, 2003, at Guntur with the aim of popularizing the subspecialty and creating voice clinics in all major cities. Several phonosurgery workshops have been organized in the country to popularize the subspecialty.

Worldwide, there is no agreed minimal training program in phonosurgery. The need for a comprehensive training was suggested as early as 1879 by Louis Elsberg at the first meeting of the American Laryngological Association.' It has also been emphasized by Sataloff in his paper "Education in Laryngology", published in $1999 .^{2}$ The nature of current training will determine the standards of the next generation of phonosurgeons and in turn shape the future of this subspecialty.

\section{Objectives}

The objective of this study was to assess exposure of postgraduate trainees in otolaryngology to phonosurgery in various colleges across the country.

\section{Materials and Methods}

A questionnaire (Appendix) was distributed to residents attending the 15th Annual Conference of Association of Phonosurgeons of India held at Kolkata in 2019 and to various Heads of ENT Departments across the country via e-mail. No personal or residency program identifying information was requested or collected, and the data are reported in aggregate only.

No incentives were offered to the residents for participation. The e-mail contained a hyperlink to the online survey. Data were extracted from online survey forms and processed by Microsoft Excel.
${ }^{1-3}$ Department of ENT Head and Neck Surgery, Vivekananda Institute of Medical Sciences, Kolkata, West Bengal, India

Corresponding Author: Shaoni D Sanyal, Department of ENT Head and Neck Surgery, Vivekananda Institute of Medical Sciences, Kolkata, West Bengal, India, Phone: +919051040747, e-mail: shaoni.sanyal@ gmail.com

How to cite this article: Sanyal SD, Roychowdhury A, Raychowdhury R. Phonosurgical Training in India: A Sample Survey. Int J Phonosurg Laryngol 2019;9(1):13-16.

Source of support: Nil

Conflict of interest: None

\section{Results}

A total of 67 trainees responded to the survey. Fifty-five were junior residents and the remaining were senior residents. The yearwise distribution of the residents is shown in Table 1. Majority of the trainees were MS candidates (53), and the rest were either DNB candidates (12) or diploma students (2). The distribution of candidates according to their training program is shown in Figure 1.

An ideal phonosurgery setup requires full-time speech and language therapy, a multidisciplinary voice clinic and stroboscopy. The availability of these in each department was assessed and the results were shown in Figure 2.

Out of 67 trainees, 48 worked in a department with a speech and language therapist (SLT). Fifteen trainees had multidisclipinary Table 1: Distribution of residents according to year of residency

\begin{tabular}{llc}
\hline & Junior residents & Senior residents \\
\hline First year & 16 & 8 \\
Second year & 14 & 2 \\
Third year & 25 & 2 \\
Total & 55 & 12 \\
\hline
\end{tabular}

OThe Author(s). 2019 Open Access This article is distributed under the terms of the Creative Commons Attribution 4.0 International License (https://creativecommons. org/licenses/by-nc/4.0/), which permits unrestricted use, distribution, and non-commercial reproduction in any medium, provided you give appropriate credit to the original author(s) and the source, provide a link to the Creative Commons license, and indicate if changes were made. The Creative Commons Public Domain Dedication waiver (http://creativecommons.org/publicdomain/zero/1.0/) applies to the data made available in this article, unless otherwise stated. 


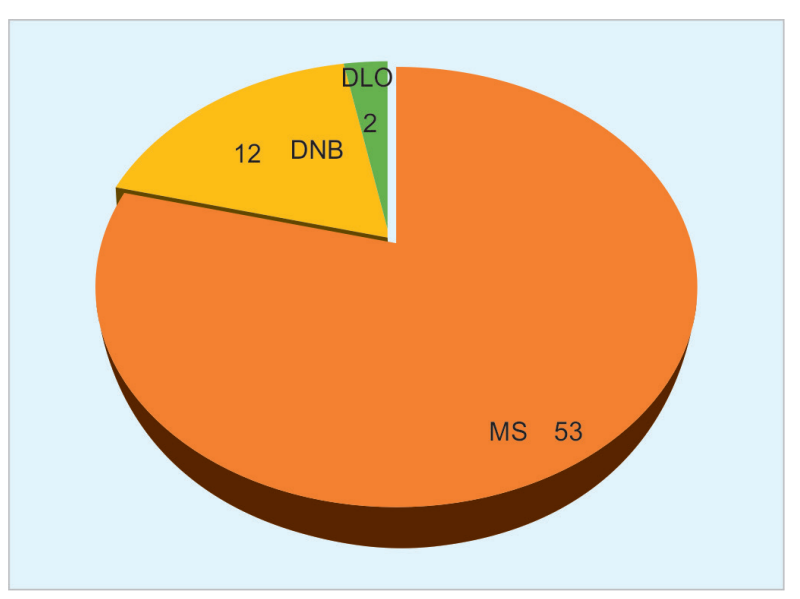

Fig. 1: Distribution of residents according to training program

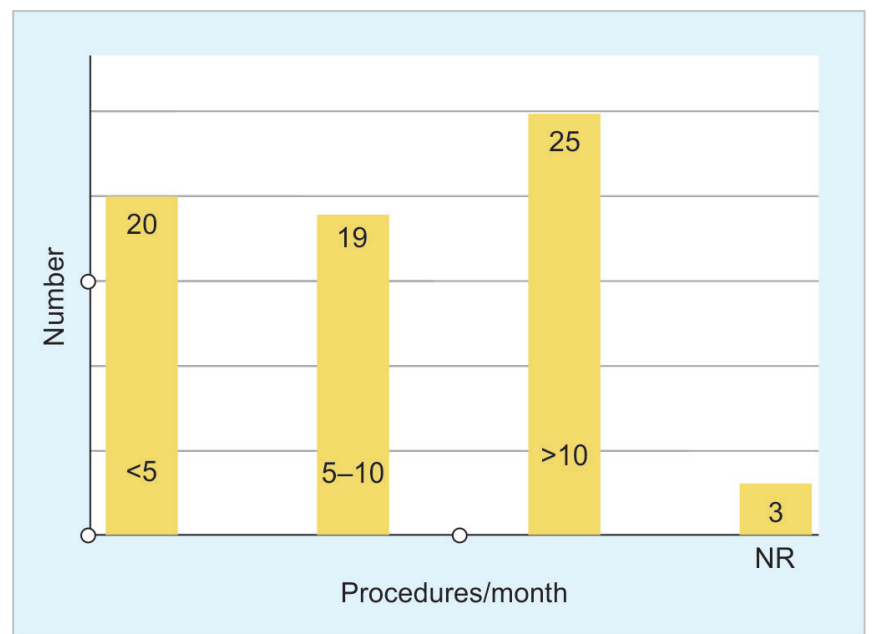

Fig. 3: Microscopic laryngeal surgery procedures performed per month

voice clinics; 17 trainees had exposure to a stroboscopy unit. Only 3 trainees worked in departments with all facilities. Of the departments with a multidisclipinary voice clinic, only $60 \%$ ran a weekly clinic. The clinic was run once every 2 weeks in $20 \%$ and once every month in $20 \%$ cases.

Most of the trainees (39) were in units performing less than 10 MLS procedures per month. Only 25 were in units performing more than $10 \mathrm{MLS}$ procedures in every month. Three trainees did not answer this question (nonrespondents) (Fig. 3).

Of the 67 respondents, 8 had performed MLS independently. Thirty-seven of the respondents had performed MLS procedures under supervision (Fig. 4)

Residents were asked about their experience in removing vocal fold lesions with the use of phonomicrosurgical techniques (i.e., microflap, etc.). Most (30) had performed excision of polyps under supervision; only 6 had performed this surgery using phonomicrosurgical technique (Fig. 5).

Thyroplasty and injection laryngoplasty were techniques which were observed by most residents ( 37 and 35 , respectively), but rarely performed. It should be noted that 5 trainees had never observed thyroplasty and 10 trainees had never observed injection laryngoplasty (Fig. 6).

Cold steel microsurgery alone was available to 37 out of 67 trainees. Thirty had some exposure to other modalities such as laser, coblation, and microdebrider (Fig. 7).

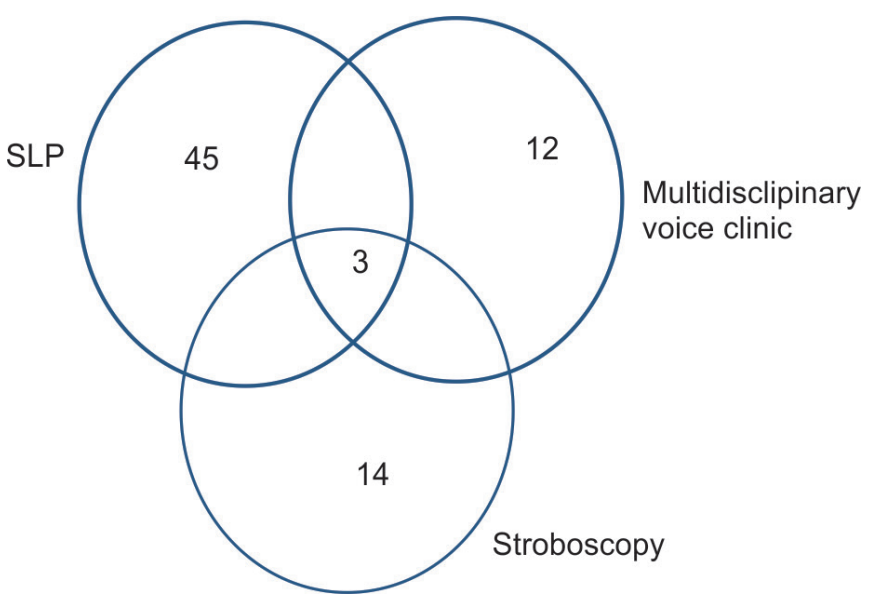

Fig. 2: Diagramatic representation of facilities available for phonosurgery

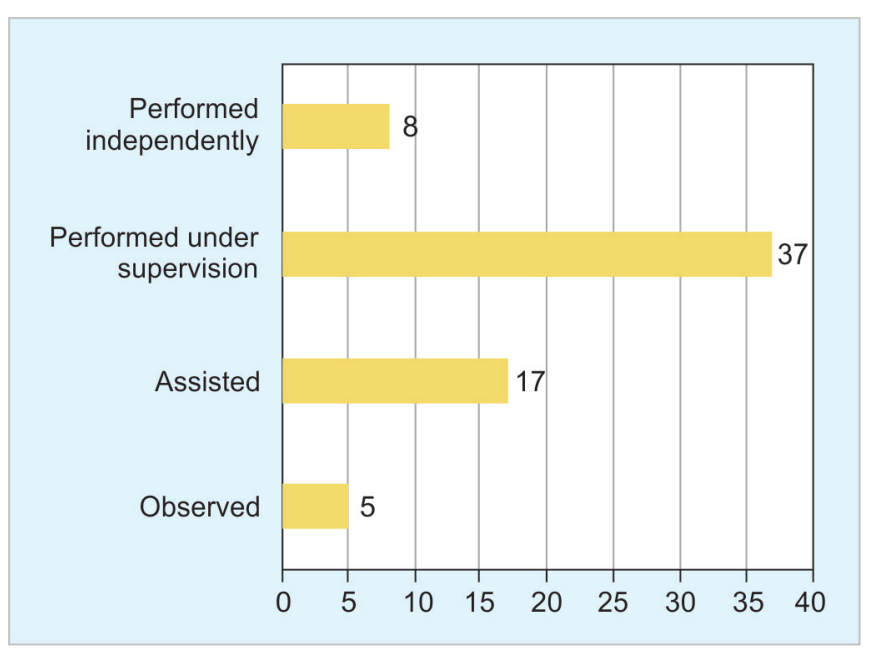

Fig. 4: Exposure to microscopic laryngeal surgeries

Follow-up of the patients ranged from 2 weeks to 6 months. Twenty-five trainees followed up patients for 2 weeks only, 18 trainees followed them up for 1 month, 23 trainees followed them up for 6 months, and 1 trainee did not answer the question (Fig. 8).

Nearly half (49\%) the respondents had never attended any phonosurgery workshop. Most of the respondents (74\%) who did attend phonosurgery workshops, participated in hands on training.

\section{Discussion}

Phonosurgery requires a good understanding of the physiology of voice production and the nature of change caused by different pathologies to the quality of voice. Besides surgery, voice rehabilitation and speech therapy play a crucial role in restoring voice.

The issue of training in laryngology was first raised by Louis Elsberg in his Presidential address to the American laryngological Association in 1879. In 1921, a 6-week course was considered to be typical duration for speciality training in otolaryngology. ${ }^{1}$

Tardy ${ }^{3}$ was among the first to highlight the importance of combining the microscope with a color television camera, in his 1972 article "Microscopic Laryngology: Teaching Techniques." He advocated television display of laryngeal surgery for the purpose of enhancing training by allowing everyone in the operating room to see what the surgeon was doing. 

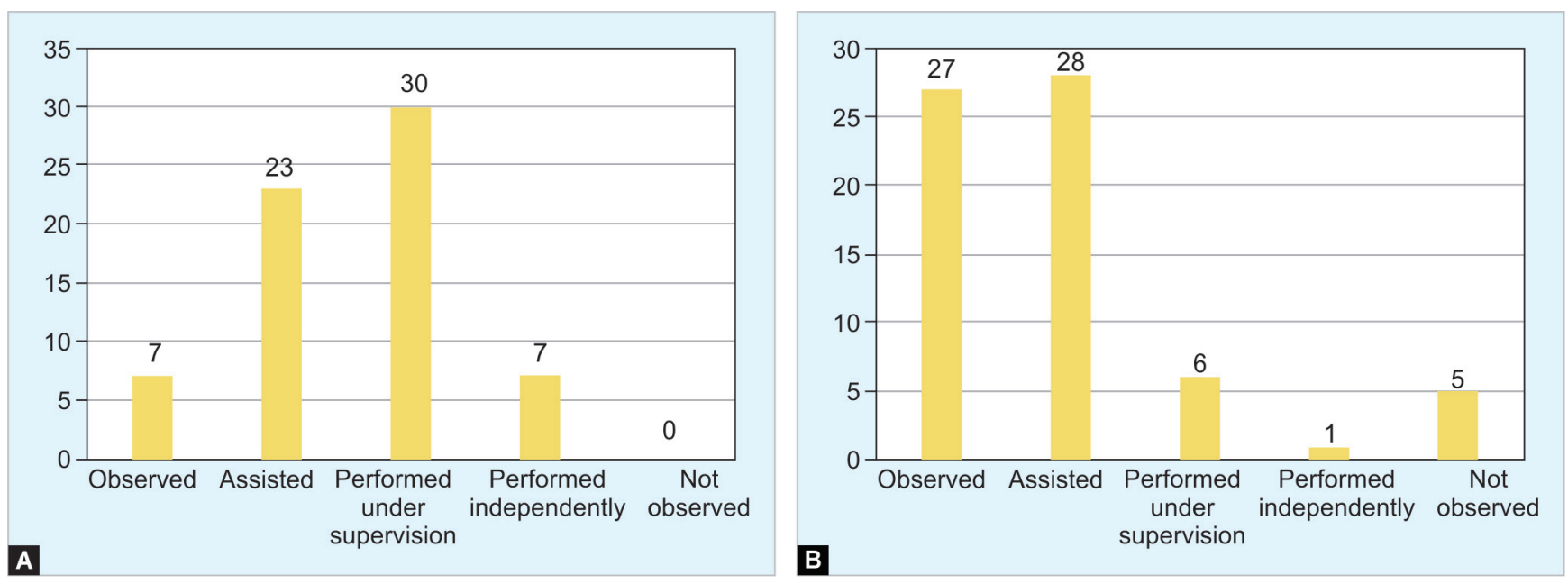

Figs 5A and B: (A) Performance of microscopic laryngeal surgery; (B) Use of Microflap technique

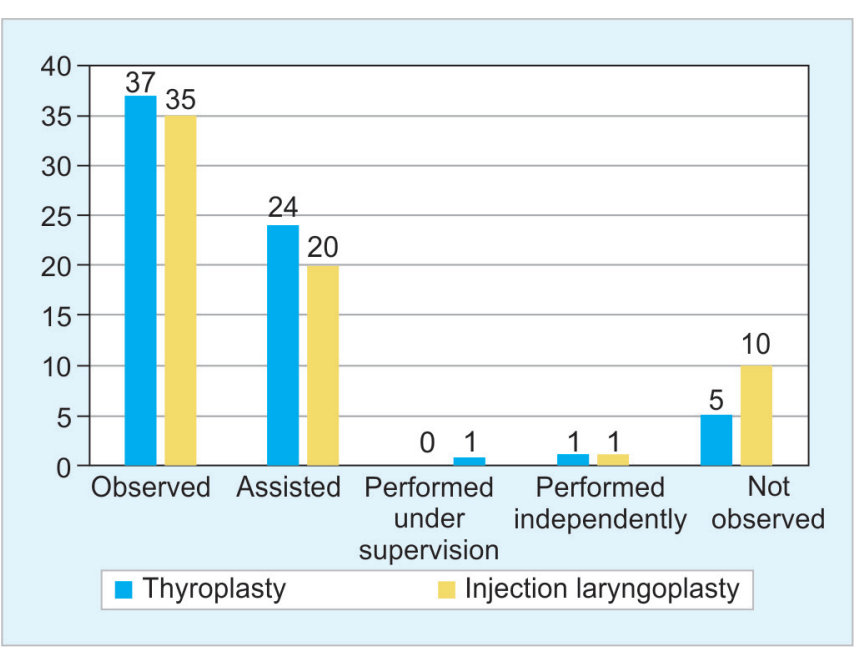

Fig. 6: Exposure to thyroplasty and injection laryngoplasty

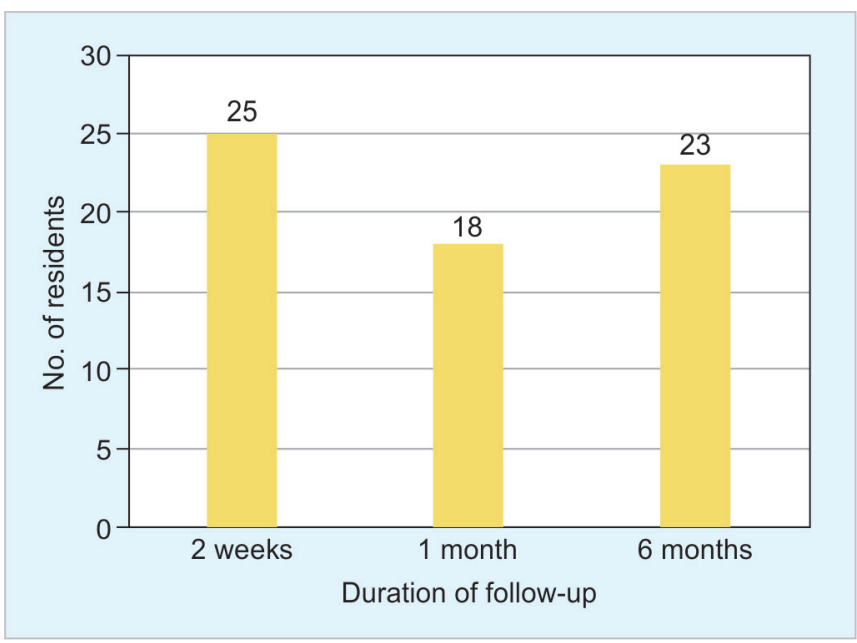

Fig. 8: Duration of follow-up in phonosurgery

Nearly five decades later, achieving a uniform level of training still remains challenging. Our study shows that of the 67 respondents, only 3 had access to all the necessary infrastructure (SLT, stroboscopy unit, and multidisciplinary voice clinic) required to treat patients. Seventy-five percent of the respondents did

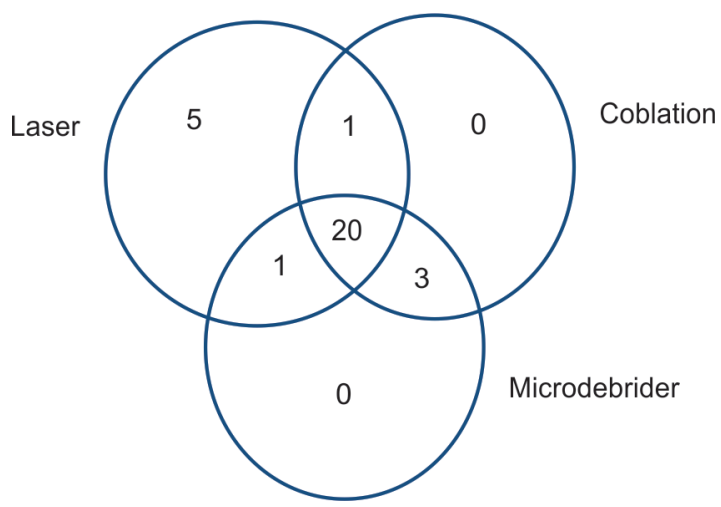

Fig. 7: Use of various modalities in phonosurgery

not have a stroboscopy unit at their institution. The exposure to phonosurgery was restricted with only $37 \%$ respondents having a case load of more than $10 \mathrm{MLS}$ per month.

This echoes with a study conducted by Shah et al. ${ }^{4}$ In his study, a survey was conducted among otolaryngological residents in USA and Canada. $60.4 \%$ of the trainees (both junior and senior residents) stated that their residency program included a rotation that was focused on laryngology. Only $18.8 \%$ of respondents overall felt "very satisfied" with their phonomicrosurgical experience during residency. The number of cases residents performed per year as primary surgeon was quite low. Among senior residents, just over $20 \%$ performed greater than 10 cases per year. This lack of operative experience is the likely cause of the low comfort level with phonomicrosurgery.

In our study, only $18 \%$ of the trainees followed up patients for a 6-month duration and evaluated their postoperative results. In the study conducted by Shah et al., $57.8 \%$ residents were not able to objectively analyse their postoperative results.

Most trainees (55\%) in our study had performed microscopic laryngeal surgery (MLS) under supervision, but only $12 \%$ were able to perform it independently. Interestingly, of the eight trainees who were confident in performing MLS independently, five were third year junior residents. This reflects a lack in standardisation of training.

\section{Conclusion}

Most trainees acquire the basic level of skill in this field by applying for fellowships after postgraduate training. The majority of trainees 
need a more thorough understanding of the basic principles of phonosurgery to effectively treat their patients. Workshops and cadaver dissections may be an effective way to impart better understanding of this field, until it can become a regular feature of postgraduate training.

Our survey shows that of the 181 departments which offer masters as well as diploma in ENT, few are fully equipped to offer modern phonosurgical assessment and management, and as a result of which, very few trainees have adequate exposure in this subspecialty. ${ }^{5}$

APSI is ideally placed to establish minimum training standards for this field. Whether these skills should be a part of 3-year postgraduate training program or should be taught during fellowship remains a matter of debate.

\section{Clinical Significance}

Few ENT departments in India are fully equipped to offer modern phonosurgical training.

\section{Appendix}

Questionnaire to assess exposure of trainees/residents to phonosurgery

Q1. What is your training program?

- MS

- DNB

Q2. What is your current level of training?

- Junior resident: 1 st year, 2 nd year, $3 r d$ year

- Senior resident: 1st year, 2nd year, 3rd year

Q3. Does your institute have a full-time speech and language therapist?

- Yes

- No

Q4. Does your institute have a stroboscopy facility?

- Yes

- No

Q5. Does your institute have a regular multidisciplinary voice clinic?

- Yes

- No

- If yes, is it

- Weekly

- Bi-weekly

- Monthly

Q6. What is your exposure to microscopic laryngeal surgery (MLS)?

- Observed

- Assisted

- Performed under supervision

- Performed independently

Q7. On average how many MLS procedures are carried out every month?

- $<5$

- $5-10$

- $>10$

Q8. What is your exposure to microscopic laryngeal surgery (MLS) for vocal nodules/vocal polyps?
Workshops and cadaver dissections may be an effective way to impart better understanding of this field.

\section{References}

1. Elsberg L. Presidential address to the first meeting of the american laryngological association. Trans American Laryngol Assoc 1879;1: 33-90.

2. Sataloff RT. Education in Laryngology: rising to old challenges. Ann Otol Rhinol Laryngol 1999;108(11):1046-1052. DOI: $10.1177 / 000348949910801105$.

3. Tardy ME. Microscopic laryngology: teaching techniques. Laryngoscope 1972;82:1315-1322. DOI: 10.1288/00005537197207000-00021.

4. Shah MD, Johns IIIMM, Statham M, et al. Assessment of phonomicrosurgical training in otolaryngology residencies: a resident survey. Laryngoscope 2013;123(6):1474-1477. DOI: 10.1002/ lary. 23763.

5. MS (ENT) Colleges in India. Targetstudy, 2019. Available from: www. targetstudy.com/colleges/ms-ent-degree-colleges-in-india.html.

- Observed

- Assisted

- Performed under supervision

- Performed independently

Q9. What is your exposure to microflap technique?

- Observed

- Assisted

- Performed under supervision

- Performed independently

Q10. What is your exposure to laryngeal framework surgery (thyroplasty)?

- Observed

- Assisted

- Performed under supervision

- Performed independently

Q11. What is your exposure to injection laryngoplasty?

- Observed

- Assisted

- Performed under supervision

- Performed independently

Q12. What instruments are used for MLS (microscopic laryngeal surgery) in your department?

- Cold steel

- Laser

- Microdebrider

- Coblation

Q13. How often are patients followed up?

- 2 weeks

- 1 month

- 6 months

- Not at all

Q14. Have you ever attended a phonosurgery workshop?

- Yes

- No

- If yes, in what capacity?

- Observer

- Hands-on 